\title{
Análise e descrição matemática da cinética de secagem de folhas de capim-limão
}

\author{
Ana P. Martinazzo ${ }^{1}$, Paulo C. Corrêa ${ }^{2}$, Osvaldo Resende ${ }^{3}$ \& Evandro de C. Melo ${ }^{2}$
}

\section{RESUMO}

As características da secagem de folhas de Cymbopogon citratus (D.C.) Stapf em camada delgada, foram estudadas para a faixa de temperatura de $30-60{ }^{\circ} \mathrm{C}$, utilizando-se modelos semi-teóricos e empíricos. O modelo de Midilli foi o que melhor descreveu as curvas de secagem. Os valores da difusividade efetiva variaram de $4 \times 10^{-12}$ a 3,9 x 10-11 $\mathrm{m}^{2} \mathrm{~s}^{-1}$ para a faixa de temperatura avaliada. Os valores obtidos da difusividade efetiva mostraram uma dependência de Arrhenius. O valor da energia de ativação encontrado foi de $63,47 \mathrm{~kJ} \mathrm{~mol}^{-1}$.

Palavras-chave: Cymbopogon citratus (D.C.) Stapf, secagem em camada delgada, modelagem matemática, difusividade efetiva

\section{Analysis and mathematical description of drying kinetic of lemon grass leaves}

\begin{abstract}
Thin layer drying characteristics of Cymbopogon citratus (D.C.) Stapf leaves were studied for the temperature range of 30-60 ${ }^{\circ} \mathrm{C}$, using semi-theoretical and empirical models. The Midilli model was found to be the best model for describing the drying curves. The effective diffusivity values ranged from $4 \times 10^{-12}$ to $3.9 \times 10^{-11} \mathrm{~m}^{2} \mathrm{~s}^{-1}$ in the studied temperature range. Calculated values of the effective diffusivity showed Arrhenius-type temperature dependence. The activation energy value found was $63.47 \mathrm{~kJ} \mathrm{~mol}^{-1}$.
\end{abstract}

Key words: Cymbopogon citratus (D.C.) Stapf, thin layer drying, mathematical modeling, effective diffusivity 


\section{INTRODUÇÃO}

A crescente procura por plantas medicinais, aromáticas e condimentares, é observada em diversos países devido à tendência dos consumidores em utilizarem, preferencialmente, produtos farmacêuticos ou alimentícios de origem natural.

O mercado mundial de fitoterápicos foi avaliado em US\$12,4 bilhões ao ano, representando 5\% do mercado mundial de produtos farmacêuticos. No Brasil, as espécies medicinais mais cultivadas e comercializadas não são nativas e grande parte foi trazida pelos imigrantes, como a camomila (Chamomilla recutita), a hortelã (Mentha spp.), o manjericão (Ocimum spp.), o capim-limão (Cymbopogon citratus) e a vinca (Catharanthus roseus) (Amaral et al., 1999; Simões et al., 2003).

O Cymbopogon citratus, popularmente conhecido como capim-limão, erva-cidreira ou capim-santo, é originário da Índia, desenvolvendo-se em todo o Brasil. Pertencente à família Poaceae, é uma erva aromática cujas folhas contêm, em sua composição química, o óleo essencial encontrado em células oleríferas; possui atividade antibacteriana, atua na diminuição da atividade motora e no aumento do período de sono; é anticonvulsionante, antiespasmódico e analgésico, sendo também utilizado como aromatizante de ambiente e como material de partida para a síntese da Vitamina A (Lorenzi \& Matos, 2002).

Nas espécies medicinais a secagem é uma operação unitária de preparação para o armazenamento a fim de atender às necessidades da indústria farmacêutica de fitoterápicos, que não tem estrutura para usar as plantas frescas nas quantidades exigidas para a produção industrial (Lorenzi \& Matos, 2002).

A conservação pela secagem se baseia no fato de que tanto os microrganismos como as enzimas e todo o mecanismo metabólico, necessitam de certa quantidade de água para suas atividades. Com a redução da água disponível, conseqüentemente serão reduzidas a atividade de água e a velocidade das reações químicas no produto, bem como o desenvolvimento de microrganismos (Christensen \& Kaufmann, 1974).

Na literatura são citados vários métodos para se analisar a secagem de produtos higroscópicos (teóricos, semi-empíricos e empíricos). Segundo Brooker et al. (1992), nos métodos teóricos normalmente se consideram as condições externas sob as quais a operação ocorre, como também os mecanismos internos de transferência de energia e massa e seus efeitos. Dependendo do material que se está secando, a umidade pode movimentar-se no seu interior, por mecanismos diferentes. Em produtos capilares porosos, como a maioria dos produtos de origem agrícola, os possíveis mecanismos de transporte de umidade, são: difusão líquida, difusão capilar, difusão na superfície, fluxo hidrodinâmico, difusão de vapor e difusão térmica.

A teoria da difusão líquida tem sido amplamente empregada na área de secagem, embora existam algumas suposições a serem consideradas para sua aplicação, como: redução do volume desprezada, não existência do efeito de capilaridade, equilíbrio térmico instantâneo com o ar e os efeitos da transferência de energia e massa de um corpo para outro, admitidos como desprezíveis. Os modelos teóricos que descrevem a taxa decrescente de secagem de um sólido consideram, geralmente, como mecanismo principal, a difusão baseada na segunda Lei de Fick, que expressa que o fluxo de massa por unidade de área é proporcional ao gradiente de concentração de água (Park et al., 2002; Romero-Peña \& Kieckbusch, 2003).

O método empírico é um método de abordagem com base em dados experimentais e na análise adimensional. Os modelos empíricos de secagem apresentam uma relação direta entre o conteúdo médio de umidade e o tempo de secagem; omitem os fundamentos do processo de secagem e seus parâmetros não têm significado físico; conseqüentemente, não oferecem uma visão apurada dos processos importantes que ocorrem durante o fenômeno, embora descrevam as curvas de secagem para determinadas condições experimentais (Keey, 1972); entre esses modelos, tem-se o de Thompson et al. (1968) e o de Wang \& Singh (1978), tradicionalmente utilizados nos estudos de secagem de produtos agrícolas e de alimentos.

Muita ênfase se tem dado ao desenvolvimento de modelos semiteóricos, que concorrem para que haja harmonia entre a teoria e a facilidade de uso. Tais modelos se baseiam, de modo geral, na Lei de Newton para resfriamento aplicada à transferência de massa. Quando se aplica esta Lei, presume-se que as condições sejam isotérmicas e que a resistência à transferência de umidade se restrinja apenas à superfície do produto (Brooker et al., 1992). Entre os modelos semiteóricos, o modelo de Dois Termos, o de Henderson e Pabis, o de Lewis, o de Page e o de Page Modificado, têm sido amplamente utilizados (Panchariya et al., 2002).

Atualmente, pesquisas relacionadas ao estudo da cinética de secagem em camada delgada, são realizadas com diversos produtos agrícolas, como sementes, grãos, frutos e em algumas espécies de plantas, com importância econômica. Observa-se, conforme o produto e as condições do processo de secagem, que diferentes modelos se ajustam a cada situação específica.

Panchariya et al. (2002) ajustaram várias equações aos dados experimentais da secagem de "chá-preto” e concluíram que o modelo de Lewis reproduziu melhor os dados experimentais de secagem em camada delgada para as faixas de temperatura de 80 a $120^{\circ} \mathrm{C}$; por sua vez, Demir et al. (2004), ao avaliarem diferentes modelos matemáticos para a secagem de louro (Laurus nobilis L.), concluíram ser o modelo de Page o que melhor descreveu o processo, enquanto Doymaz et al. (2006), avaliando a secagem de folhas de endro (Anethum graveolens L.) e de salsinha (Petroselinum crispum L.), definiram o modelo de Midilli como o mais adequado para descrever as curvas de secagem nas temperaturas de 40 a $70{ }^{\circ} \mathrm{C}$.

Ante o exposto objetivou-se, através do presente trabalho, ajustar modelos matemáticos de secagem em camada delgada, aos dados experimentais obtidos na secagem de folhas de capim-limão, Cymbopogon citratus (D.C.) Stapf, em diferentes temperaturas do ar de secagem. 


\section{MATERIAL E MÉTODOS}

Este estudo foi realizado no Laboratório de Propriedades Físicas e Avaliação da Qualidade, pertencente ao CENTREINAR, localizado na Universidade Federal de Viçosa, MG; nele, utilizaram-se folhas de capim-limão (Cymbopogon citratus), colhidas manualmente, com teor de água inicial de aproximadamente 3,55 b.s.

Depois da colheita, antes de se iniciarem os processos de secagem, o material foi transportado para local apropriado, onde as folhas foram selecionadas, retirando-lhes as partes doentes e danificadas, assim como qualquer parte de outro vegetal ou material estranho, e cortadas manualmente no comprimento de $30 \mathrm{~cm}$. Os teores de água do produto foram determinados pelo método da estufa, a $105 \pm 1{ }^{\circ} \mathrm{C}$, até peso constante, em três repetições.

Realizaram-se as secagens utilizando-se um secador de bandejas com temperaturas controladas (30, 40, 50 e $60{ }^{\circ} \mathrm{C}$ ) e umidade relativa do ar monitorada, através de um psicrômetro. A umidade relativa do ar secante era calculada por meio de um programa computacional desenvolvido a partir de equações psicrométricas. Durante a operação de secagem foram realizadas pesagens periódicas, até se atingir o teor de água final de 0,12 b.s., enquanto as curvas de secagem foram ajustadas aos dados experimentais utilizando-se treze diferentes equações empíricas e semi-empíricas, descriminadas a seguir:

Modelo de Newton

$$
\mathrm{RU}=\exp (-\mathrm{k} \cdot \mathrm{t})
$$

Modelo de Page

$$
\mathrm{RU}=\exp \left(-\mathrm{k} \cdot \mathrm{t}^{\mathrm{n}}\right)
$$

Modelo de Page Modificado

$$
\mathrm{RU}=\exp \left[-(\mathrm{k} \cdot \mathrm{t})^{\mathrm{n}}\right]
$$

Modelo de Thompson

$$
\mathrm{t}=\mathrm{a} \cdot \ln (\mathrm{RU})+\mathrm{b} \cdot[\ln (\mathrm{RU})]^{2}
$$

Modelo de Difusão (Lei de Fick)

$$
\mathrm{RU}=\frac{\mathrm{U}-\mathrm{Ue}}{\mathrm{Ui}-\mathrm{Ue}}=\frac{8}{\pi^{2}} \sum_{n=0}^{\infty} \frac{1}{(2 n+1)^{2}} \exp \left[-(2 n+1)^{2} \pi^{2} \mathrm{D} \frac{\mathrm{t}}{4 \mathrm{~L}^{2}}\right]
$$

Modelo de Henderson e Pabis

$$
\mathrm{RU}=\mathrm{a} \cdot \exp (-\mathrm{k} . \mathrm{t})
$$

Modelo de Logarítmico

$$
\mathrm{RU}=\mathrm{a} \cdot \exp (-\mathrm{k} \cdot \mathrm{t})+\mathrm{c}
$$

Modelo de Dois termos

$$
R U=a \cdot \exp \left(-k_{0} \cdot t\right)+b \cdot \exp \left(-k_{1} \cdot t\right)
$$

Modelo de Exponencial de dois termos

$$
R U=a \cdot \exp (-k \cdot t)+(1-a) \exp (-k \cdot a \cdot t)
$$

Modelo de Wang e Sing

$$
\mathrm{RU}=1+\mathrm{at}+\mathrm{bt}^{2}
$$

Modelo de Henderson \& Pabis modificado

$$
\mathrm{RU}=\mathrm{a} \cdot \exp (-\mathrm{k} \cdot \mathrm{t})+\mathrm{b} \cdot \exp \left(-\mathrm{k}_{\mathrm{o}} \cdot \mathrm{t}\right)+\mathrm{c} \cdot \exp \left(-\mathrm{k}_{1} \cdot \mathrm{t}\right)
$$

Modelo de Midilli et al.

$$
\mathrm{RU}=\mathrm{a} \cdot \exp \left(-\mathrm{k} \cdot \mathrm{t}^{\mathrm{n}}\right)+\mathrm{b} \cdot \mathrm{t}
$$

Modelo de Aproximação da difusão

$$
R U=\mathrm{a} \cdot \exp (-\mathrm{k} \cdot \mathrm{t})+(1-\mathrm{a}) \exp (-\mathrm{k} \cdot \mathrm{b} \cdot \mathrm{t})
$$

em que:

RU - razão de umidade do produto, adimensional

$\mathrm{t}$ - tempo de secagem, $\mathrm{s}$

$\mathrm{k}, \mathrm{k}_{0}, \mathrm{k}_{1}$ - coeficientes de secagem, $\mathrm{s}^{-1}$

a, b, c, $\mathrm{n}$ - constantes dos modelos

$n$ - número de termos da equação

$\mathrm{D}$ - coeficiente de difusão, $\mathrm{m}^{2} \mathrm{~s}^{-1}$

$\mathrm{L}$ - espessura do produto, $\mathrm{m}$

Para o cálculo da razão de umidade (RU), durante as secagens nas diferentes temperaturas, utilizou-se a expressão:

$$
R U=\frac{U-U_{e}}{U_{i}-U_{e}}
$$

em que:

U - teor de água do produto, decimal b.s.

$\mathrm{U}_{\mathrm{i}}$ - teor de água inicial do produto, decimal b.s.

$\mathrm{U}_{\mathrm{e}}$ - teor de água de equilíbrio do produto, decimal b.s.

Determinou-se a umidade de equilíbrio higroscópico (Ue) pela Eq. 15, proposta por Corrêa et al. (2002), para plantas medicinais, com seus parâmetros determinados pelo processo de dessorção.

$$
\mathrm{U}_{\mathrm{e}}=\frac{1}{\left(\mathrm{aT} \mathrm{T}^{\mathrm{b}}+\mathrm{UR}^{\mathrm{c}}\right)}
$$

em que:

$\mathrm{T}$ - temperatura do ar, ${ }^{\circ} \mathrm{C}$

UR - umidade relativa do ar, decimal

a, b, c - constantes que dependem da natureza do produto. No caso do capim-limão: $a=-1,0484$; $\mathrm{b}=-0,0221$ e $\mathrm{c}=-0,0628$.

Para o ajuste dos modelos matemáticos aos dados experimentais de secagem, realizou-se análise de regressão não linear, pelo método Simplex e Quasi-Newton, através do programa computacional STATISTICA 5.0 ${ }^{\circledR}$.

O grau de ajuste do modelo aos dados experimentais foi avaliado em função da magnitude do coeficiente de determinação ajustado $\left(\mathrm{R}^{2}\right)$ e do erro médio estimado (SE).

$$
\mathrm{SE}=\sqrt{\frac{\sum\left(\mathrm{Y}-\mathrm{Y}_{0}\right)^{2}}{\mathrm{GLR}}}
$$

em que:

Y - valor observado experimentalmente

$\mathrm{Y}_{0}$ - valor calculado pelo modelo

GLR - graus de liberdade do modelo

A dependência da difusividade efetiva (D) com a temperatura foi analisada pela equação de Arrhenius: 


$$
\mathrm{D}=\operatorname{Aexp}\left(-\frac{\mathrm{E}}{\mathrm{RT}}\right)
$$

em que:

$$
\begin{aligned}
& \text { A - constante, } \mathrm{m}^{2} \mathrm{~s}^{-1} \\
& \mathrm{E} \text { - energia de ativação, } \mathrm{J} \mathrm{mol}^{-1} \\
& \mathrm{R} \text { - constante universal dos gases, } 8,314 \mathrm{~J} \mathrm{~mol}^{-1} \mathrm{~K}^{-1} \\
& \mathrm{~T} \text { - temperatura absoluta, } \mathrm{K}
\end{aligned}
$$

\section{RESULTADOS E DISCUSSÃO}

Apresentam-se na Figura 1, as curvas de secagem em camada delgada de folhas de $C$. citratus em diferentes temperaturas. Para facilitar a visualização não apenas desta Figura mas, também, das demais, o tempo foi referido em horas, apesar de toda a análise estatística considerar o tempo em minutos.

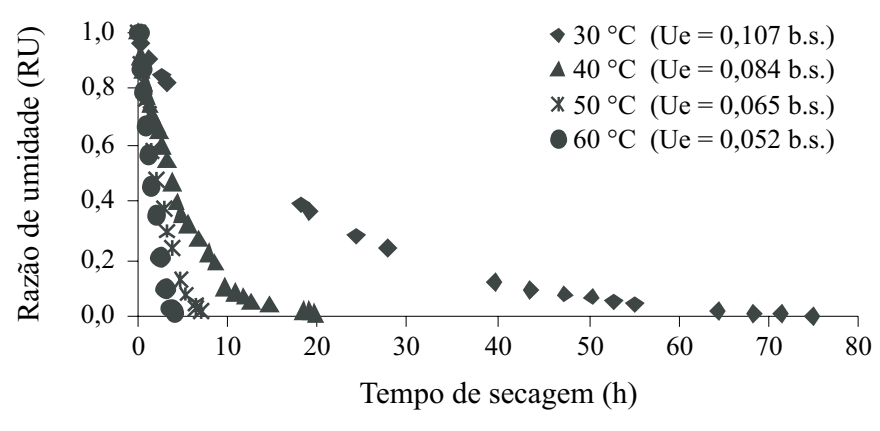

Figura 1. Variações da razão de umidade em função do tempo para diferentes temperaturas do ar de secagem

Observa-se, na Figura 1, a influência da temperatura do ar na cinética do processo. Como previsto, o tempo de secagem decresce com o aumento da temperatura do ar; para se alcançar o teor de água em torno de 0,12 b.s., o tempo necessário foi de 4, 7, 20 e 75 h para as temperaturas de 60, 50 , 40 e $30^{\circ} \mathrm{C}$, respectivamente, cujos resultados estão de acordo com estudos realizados anteriomente para a secagem de diversos produtos alimentícios (Madamba et al., 1996; Davidson et al., 2004; Mohapatra \& Rao, 2005)
Apresenta-se, na Tabela 1, o resumo do ajuste dos modelos por meio de regressão não linear aos dados experimentais de secagem de folhas de $C$. citratus em camada fina, considerando-se as diferentes temperaturas do ar de secagem.

Dentre os modelos avaliados, a equação de Midilli et al. foi a que melhor se ajustou aos dados observados de razão de umidade. Enquanto SE variou entre 0,0073 a 0,1013 para todos os modelos analisados, este valor oscilou entre 0,0073 e 0,0248 para o modelo de Midilli et al., nas diferentes condições experimentais. Em relação ao coeficiente de determinação $\left(R^{2}\right)$, a equação apresentou valores elevados estando, para todos os tratamentos, acima de $98 \%$ o que, segundo Madamba et al. (1996) significa um bom ajuste dos modelos para representação do fenômeno de secagem. Os valores dos parâmetros obtidos em cada modelo estão apresentados na Tabela 2 para as diferentes condições experimentais.

Com base nos valores apresentados na Tabela 2, realizou-se ajuste de equações para determinação dos valores dos parâmetros do modelo de Midilli et al., em função da temperatura do ar de secagem. Na Tabela 3 têm-se as equações para estimativa dos parâmetros do modelo, com os respectivos coeficientes de determinação $\left(\mathrm{R}^{2}\right)$ e erro médio estimado (SE).

Encontram-se, na Figura 2, os dados experimentais e calculados de razão de umidade (RU) empregando-se a equação de Midilli et al. com os parâmetros estimados por meio das equações e dos valores apresentados na Tabela 3. A Figura possibilita verificar o bom ajuste deste modelo na descrição dos resultados experimentais.

Em virtude do mecanismo de transporte da água no interior do produto ocorrer por meio de difusão de vapor, utilizou-se a Eq. 5, baseada na Lei de Fick, para produtos com forma geométrica de placa plana, para calcular, a partir dos dados experimentais, a difusividade efetiva do produto (D). Os valores encontrados para cada condição de secagem estão descritos na Tabela 4.

Observa-se que os valores da difusividade aumentaram com a elevação da temperatura de secagem. Doymaz et al.

\begin{tabular}{|c|c|c|c|c|c|c|c|c|}
\hline \multirow{2}{*}{ Modelos matemáticos } & \multicolumn{4}{|c|}{$R^{2}(\%)$} & \multicolumn{4}{|c|}{ SE } \\
\hline & 30 & 40 & 50 & 60 & 30 & 40 & 50 & 60 \\
\hline Exponencial & 99,86 & 99,44 & 98,28 & 97,25 & 0,0140 & 0,0244 & 0,0452 & 0,0573 \\
\hline Page & 99,87 & 99,44 & 99,04 & 99,17 & 0,0141 & 0,0249 & 0,0338 & 0,0333 \\
\hline Page Modificado & 99,87 & 99,44 & 99,04 & 99,17 & 0,0141 & 0,0249 & 0,0338 & 0,0003 \\
\hline Thompson & 99,86 & 99,44 & 99,04 & 97,25 & 0,0144 & 0,0249 & 0,0338 & 0,0604 \\
\hline Difusão Oito Termos & 98,10 & 97,08 & 94,09 & 91,40 & 0,0515 & 0,0550 & 0,0077 & 0,1013 \\
\hline Henderson e Pabis & 99,90 & 99,49 & 98,33 & 97,66 & 0,0124 & 0,0238 & 0,0416 & 0,0558 \\
\hline Logarítmico & 99,96 & 99,59 & 99,64 & 99,61 & 0,0082 & 0,0218 & 0,0206 & 0,0241 \\
\hline Dois termos & 99,92 & 99,47 & 98,33 & 97,66 & 0,0113 & 0,0253 & 0,0445 & 0,0632 \\
\hline Exponencial dois termos & 99,86 & 99,47 & 98,26 & 97,22 & 0,0143 & 0,0243 & 0,0453 & 0,0607 \\
\hline Wang e Sing & 98,13 & 96,88 & 97,86 & 98,76 & 0,0526 & 0,0587 & 0,0503 & 0,0406 \\
\hline Henderson e Pabis & 99,92 & 99,51 & 98,36 & 99,39 & 0,0121 & 0,0256 & 0,0440 & 0,0382 \\
\hline Midilli et al. & 99,97 & 99,60 & 99,63 & 99,64 & 0,0073 & 0,0219 & 0,0209 & 0,0248 \\
\hline Aproximação Difusão & 99,92 & 99,47 & 99,17 & 99,15 & 0,0111 & 0,0248 & 0,0314 & 0,0356 \\
\hline
\end{tabular}
(2006) em estudo sobre a secagem de folhas de endro (Anethum graveolens L.), encontraram valores de 6,693 x 10-10,

Tabela 1. Valores do coeficiente de determinação $\left(\mathrm{R}^{2}\right)$ e do erro médio estimado (SE), calculados para verificação do ajuste dos modelos matemáticos aos valores experimentais da secagem de folhas de $\mathrm{C}$. citratus, obtidos nas temperaturas de $30,40,50$ e $60{ }^{\circ} \mathrm{C}$ 
Tabela 2. Parâmetros obtidos dos modelos ajustados aos dados de secagem de folhas de Cymbopogon citratus, no comprimento de corte de $30 \mathrm{~cm}$ para diversas temperaturas do ar

\begin{tabular}{|c|c|c|c|c|c|c|c|}
\hline $\begin{array}{c}\text { Modelos } \\
\text { matemáticos }\end{array}$ & $\mathrm{T}\left({ }^{\circ} \mathrm{C}\right)$ & k & & & & & \\
\hline \multirow{5}{*}{ Exponencial } & 30 & 0,0009 & & & & & \\
\hline & 40 & 0,0034 & & & & & \\
\hline & 50 & 0,0061 & & & & & \\
\hline & 60 & 0,0099 & & & & & \\
\hline & $\mathrm{T}\left({ }^{\circ} \mathrm{C}\right)$ & k & n & & & & \\
\hline \multirow{5}{*}{ Page } & 30 & 0,0011 & 0,9766 & & & & \\
\hline & 40 & 0,0032 & 1,0081 & & & & \\
\hline & 50 & 0,0023 & 1,1902 & & & & \\
\hline & 60 & 0,0025 & 1,2982 & & & & \\
\hline & $\mathrm{T}\left({ }^{\circ} \mathrm{C}\right)$ & k & $n$ & & & & \\
\hline \multirow{5}{*}{$\begin{array}{c}\text { Page } \\
\text { Modificado }\end{array}$} & 30 & 0,0089 & 0,0972 & & & & \\
\hline & 40 & 0,0034 & 1,0085 & & & & \\
\hline & 50 & 0,0061 & 1,1902 & & & & \\
\hline & 60 & 0,0099 & 1,2990 & & & & \\
\hline & $\mathrm{T}\left({ }^{\circ} \mathrm{C}\right)$ & a & b & & & & \\
\hline \multirow{5}{*}{ Thompson } & 30 & $-9997,9$ & 2,9838 & & & & \\
\hline & 40 & $-3973,5$ & 3,6703 & & & & \\
\hline & 50 & 0,0061 & 1,1902 & & & & \\
\hline & 60 & $-9997,8$ & 9,9506 & & & & \\
\hline & $\mathrm{T}\left({ }^{\circ} \mathrm{C}\right)$ & D & & & & & \\
\hline \multirow{5}{*}{$\begin{array}{c}\text { Difusão } \\
\text { Oito Termos }\end{array}$} & 30 & $4 \times 10^{-5}$ & & & & & \\
\hline & 40 & $1,2 \times 10^{-5}$ & & & & & \\
\hline & 50 & $2,4 \times 10^{-5}$ & & & & & \\
\hline & 60 & $3,9 \times 10^{-5}$ & & & & & \\
\hline & $\mathrm{T}\left({ }^{\circ} \mathrm{C}\right)$ & a & k & & & & \\
\hline \multirow{5}{*}{$\begin{array}{l}\text { Henderson } \\
\text { e Pabis }\end{array}$} & 30 & 0,9790 & 0,0009 & & & & \\
\hline & 40 & 0,9822 & 0,0033 & & & & \\
\hline & 50 & 1,0181 & 0,0063 & & & & \\
\hline & 60 & 1,0499 & 0,0105 & & & & \\
\hline & $\mathrm{T}\left({ }^{\circ} \mathrm{C}\right)$ & a & k & C & & & \\
\hline \multirow{5}{*}{ Logarítmico } & 30 & 1,0005 & 0,0008 & $-0,0216$ & & & \\
\hline & 40 & 1,0038 & 0,0031 & $-0,0301$ & & & \\
\hline & 50 & 1,1954 & 0,0041 & $-0,2131$ & & & \\
\hline & 60 & 1,3391 & 0,0060 & $-0,3353$ & & & \\
\hline & $\mathrm{T}\left({ }^{\circ} \mathrm{C}\right)$ & a & k & b & $k_{1}$ & & \\
\hline \multirow{5}{*}{ Dois termos } & 30 & 0,0281 & 2,4341 & 0,9766 & 0,0009 & & \\
\hline & 40 & 0,0098 & 3,6787 & 0,9892 & 0,0034 & & \\
\hline & 50 & 1,0022 & 0,0063 & 0,0160 & 0,0063 & & \\
\hline & 60 & 0,3153 & 0,0105 & 0,7342 & 0,0105 & & \\
\hline & $\mathrm{T}\left({ }^{\circ} \mathrm{C}\right)$ & a & k & & & & \\
\hline \multirow{5}{*}{$\begin{array}{l}\text { Exponencial } \\
\text { Dois Termos }\end{array}$} & 30 & 1,2755 & 0,0009 & & & & \\
\hline & 40 & 1,3799 & 0,0038 & & & & \\
\hline & 50 & 0,0020 & 3,0190 & & & & \\
\hline & 60 & 0,0016 & 6,3278 & & & & \\
\hline & $\mathrm{T}\left({ }^{\circ} \mathrm{C}\right)$ & a & b & & & & \\
\hline \multirow{5}{*}{ Wang e Sing } & 30 & $-0,0006$ & $8,1 \times 10^{-8}$ & & & & \\
\hline & 40 & $-0,0023$ & $1,0 \times 10^{-6}$ & & & & \\
\hline & 50 & $-0,0039$ & $3,0 \times 10^{-6}$ & & & & \\
\hline & 60 & $-0,0081$ & $1,6 \times 10^{-5}$ & & & & \\
\hline & $\mathrm{T}\left({ }^{\circ} \mathrm{C}\right)$ & a & k & b & $k_{0}$ & C & $k_{1}$ \\
\hline \multirow{5}{*}{$\begin{array}{l}\text { Henderson } \\
\text { e Pabis } \\
\text { Modificado }\end{array}$} & 30 & 0,9767 & 0,0008 & 0,6384 & 2,3666 & $-0,6103$ & 1,8889 \\
\hline & 40 & $-1,8730$ & 4,2729 & 0,9767 & 0,0033 & 1,8966 & 1,4407 \\
\hline & 50 & 1,0287 & 0,0063 & $-0,9825$ & 2,4524 & 0,9538 & 2,1218 \\
\hline & 60 & 18,556 & 0,0197 & 0,1231 & 3,081 & $-17,679$ & 0,0207 \\
\hline & $\mathrm{T}\left({ }^{\circ} \mathrm{C}\right)$ & a & k & $n$ & B & & \\
\hline \multirow{5}{*}{ Midilli et al. } & 30 & 0,9739 & 0,0007 & 1,0320 & $-4 \times 10^{-6}$ & & \\
\hline & 40 & 0,9588 & 0,0021 & 1,0716 & $-1 \times 10^{-5}$ & & \\
\hline & 50 & 0,9702 & 0,0031 & 1,0949 & $-0,00235$ & & \\
\hline & 60 & 0,9898 & 0,0043 & 1,1362 & $-5,2 \times 10^{-4}$ & & \\
\hline & $\mathrm{T}\left({ }^{\circ} \mathrm{C}\right)$ & a & k & b & & & \\
\hline \multirow{4}{*}{$\begin{array}{c}\text { Aproximação } \\
\text { Difusão }\end{array}$} & 30 & 0,0208 & 1,011 & 0,0009 & & & \\
\hline & 40 & 1,2781 & 0,0037 & 1,4185 & & & \\
\hline & 50 & 186,60 & 0,0102 & 1,0032 & & & \\
\hline & 60 & 199,69 & 0,0183 & 1,0037 & & & \\
\hline
\end{tabular}

Tabela 3. Parâmetros do modelo de Midilli et al. para cálculo da razão de umidade (RU) de Cymbopogon citratus, em função da temperatura do ar de secagem $\left(\mathrm{T}-{ }^{\circ} \mathrm{C}\right)$

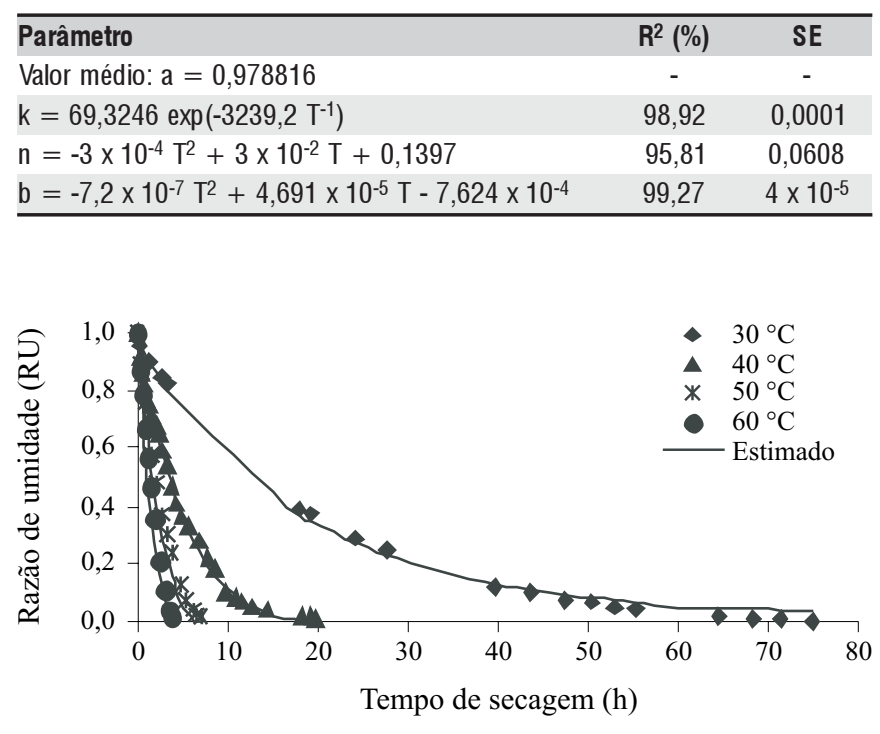

Figura 2. Valores experimentais e estimados de razão de umidade pela estimativa dos parâmetros da equação de Midilli et al.

Tabela 4. Valores da difusividade efetiva (D) obtidos para folhas de C. citratus em diferentes temperaturas do ar de secagem

\begin{tabular}{cc}
\hline Temperatura $\left({ }^{\circ} \mathrm{C}\right)$ & Difusividade $\left(\mathbf{m}^{2} \mathbf{s}^{-1}\right)$ \\
30 & $4,00 \times 10^{-12}$ \\
40 & $1,20 \times 10^{-11}$ \\
50 & $2,40 \times 10^{-11}$ \\
60 & $3,90 \times 10^{-11}$ \\
\hline
\end{tabular}

$9,205 \times 10^{-10}$ e $1,434 \times 10^{-9} \mathrm{~m}^{2} \mathrm{~s}^{-1}$ para as temperaturas de 50 , 60 e $70^{\circ} \mathrm{C}$, respectivamente. Akpinar (2006) obteve valores entre $4,53 \times 10^{-12}$ a $7,04 \times 10^{-12} \mathrm{~m}^{2} \mathrm{~s}^{-1}$ para as espécies menta (Mentha sp.), salsinha (Petroselinum crispum) e manjericão (Ocimum basilicum). Segundo Zogzas et al. (1996), os valores de $\mathrm{D}$ para produtos alimentícios se encontram na faixa de $10^{-11}$ a $10^{-9} \mathrm{~m}^{2} \mathrm{~s}^{-1}$.

A Figura 3 mostra os valores de D apresentados na forma de "In D" descritos em função do recíproco da temperatura absoluta $(1 / \mathrm{T})$. A reta obtida indica a uniformidade de variação da difusividade com a temperatura.

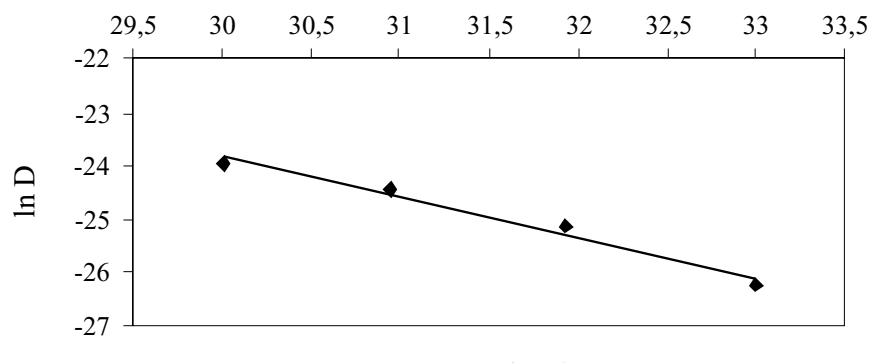

$1 / \mathrm{T} \times 10^{-4}\left(\mathrm{~K}^{-1}\right)$

Figura 3. Representação de Arrhenius para a relação entre a difusividade efetiva e a temperatura absoluta na secagem de folhas de $C$. citratus 
A energia de ativação (E) calculada da reta obtida na Figura 3, foi de $63,47 \mathrm{~kJ} \mathrm{~mol}^{-1}$, valor este próximo ao obtido por Doymaz (2006) para folhas de Mentha spicata L. (62,96 kJ mol-1).

\section{CONCLUSÕES}

1. A cinética de secagem das folhas de Cymbopogon citratus (DC.) Stapf é função direta da temperatura do ar.

2. Para obtenção do produto com teor de água de 0,12 b.s. foi gasto o tempo de $75 \mathrm{~h}$ para $30^{\circ} \mathrm{C} ; 20 \mathrm{~h}$ para $40^{\circ} \mathrm{C}$; $7 \mathrm{~h}$ para $50^{\circ} \mathrm{C}$ e de $4 \mathrm{~h}$ para $60{ }^{\circ} \mathrm{C}$.

3. O modelo matemático de secagem em camada fina, proposto por Midilli et al., foi o que melhor se ajustou aos dados experimentais.

4. Os valores do coeficiente de difusão aumentaram de acordo com a elevação da temperatura do ar de secagem, apresentando resultados entre $1,20 \times 10^{-11}$ a $4,00 \times 10^{-12} \mathrm{~m}^{2} \mathrm{~s}^{-1}$.

5. A dependência da difusividade em relação à temperatura foi descrita pela equação de Arrhenius, por meio da qual se obteve o valor da energia de ativação de $63,47 \mathrm{~kJ} \mathrm{~mol}^{-1}$.

\section{LITERATURA CITADA}

Akpinar, E. K. Mathematical modelling of thin layer drying process under open sun of some aromatic plants. Journal of Food Engineering, London, v.77, n.4, p.864-870, 2006.

Amaral, C. L. F.; Oliveira, J. E. Z.; Casali, V. W. D. Plantas medicinais e aromáticas: Melhoramento genético. Viçosa: UFV, 1999. 153p.

Brooker, D. B.; Baker-Arkema, F. W.; Hall, C. W. Drying and storage of grains and oilseeds. New York: AVI Book, 1992. 450p.

Christensen, C. M.; Kaufmann, H. H. Microflora. In: Christensen, C. M. Storage of cereal grain and their products. St. Paul: American Association of Cereals Chemists, 1974. p.158-192.

Corrêa, P. C.; Afonso Jr., P. C.; Martins, P. M.; Melo, E. C.; Radunz, L. L. Modelo matemático para representação da higroscopicidade de plantas medicinais. Revista Brasileira de Armazenamento, Viçosa, v.27, n.1, p.9-13, 2002.

Davidson, V. J.; Li, X.; Brown, R. B. Forced-air drying of ginseng root: 1. Effects of air temperature on quality. Journal of Food Engineering, Davis, v.63, n.4, p.361-367, 2004.
Demir, V.; Gunhan, T.; Yagcioglu, A. K.; Degirmencioglu, A. Mathematical modelling and the determination of some quality parameters of air-dried bay leaves. Biosystems Engineering, Edinburgh, v.88, n.3, p.325-335, 2004.

Doymaz, I. Thin-layer drying behaviour of mint leaves. Journal of Food Engineering, Davis, v.74, n.3, p.370-375, 2006.

Doymaz, I.; Tugrul, N.; Pala, M. Drying characteristics of dill and parsley leaves. Journal of Food Engineering, Davis, v.77, n.3, p.559-565, 2006.

Keey, R. B. Drying: Principles and practice. New York: Pergamon Press. 1972. 358p.

Lorenzi, H.; Matos, F. J. A. Plantas medicinais no Brasil: Nativas e exóticas. Nova Odessa: Instituto Plantarum, 2002. 512p.

Madamba, P. S.; Driscoll, R. H.; Buckle, K. A. Thin layer drying characteristics of garlic slices. Journal of Food Engineering, Davis, v.29, n.1, p.75-97, 1996.

Mohapatra, D.; Rao, P. S. A thin layer drying model of parboiled wheat. Journal of Food Engineering, Davis, v.66, n.4, p.513-518, 2005.

Panchariya, P. C.; Popovic, D.; Sharma, A. L. Thin-layer modeling of black tea drying process. Journal of Food Engineering, Davis, v.52, n.4, p.349-357, 2002.

Park, K. J.; Vohnikoza, Z.; Brod, F. P. R. Evaluation of drying parameters and desorption isotherms of garden mint leaves (Mentha crispa L.). Journal of Food Engineering, Davis, v.51, n.3, p.193-199, 2002.

Romero-Peña, L. M.; Kieckbusch, T. G. Influência de condições de secagem na qualidade de fatias de tomate. Brazilian Journal of Food Technology, Campinas, v.6, n.1, p.69-76, 2003.

Simões, C. M. O.; Schenkel, E. P.; Gosmann, G.; Mello, J. C. P. de; Mentz, L. A.; Petrovick, P. R. Farmacognosia: da planta ao medicamento. 5.ed. Porto Alegre/Florianópolis: UFRGS/UFSC, 2003. 1102p.

Thompson, T. L.; Peart, R. M.; Foster, G. H. Mathematical simulation of corn drying: A new model. Transactions of ASAE, St. Joseph, v.11, n.4, p.582-586, 1968.

Wang, C. Y.; Singh, R. P. Use of variable equilibrium moisture content in modeling rice drying. Transaction of ASAE, St. Joseph, v.11, ASAE Paper n.78-3001, 1978.

Zogzas, N. P.; Mauroulis, Z. B.; Marinos-Kouris, D. Moisture diffusivity data compilation in foodstuffs. Drying Technology, Bangkok, v.14, n.10, p.2225-2253, 1996. 